mid-2000s, but since then, increases have been more modest. These trends coincided with changes in sexual health policy and practise in Britain, resulting in greater STI testing and the use of more sensitive diagnostic tests, making STI surveillance data less indicative of risk behaviour. This paper reviews a range of evidence to examine whether, and if so how, sexual behaviour has changed in Britain since the start of the millennium.

Methods Analyses of routine data (including STI surveillance data, census data), general population surveys (including Natsal, Health Survey for England 2010 (HSE-2010), British Social Attitudes surveys), and community surveys of men-who-have-sex-with-men (MSM, including London's Gay Men's Sexual Health Surveys).

Results Demographic trends support the limited sexual behaviour data collected experimentally by HSE-2010 suggesting that increases in heterosexual risk behaviour observed between 1990 and 2000 have not been sustained since 2000. At the same time, there has been increasing tolerance in Britain of more diverse sexual lifestyles, with public attitudes towards homosexuality increasingly liberal. While the population prevalence of recent same-sex behaviour in 2010 remains around $2-3 \%$, among MSM, the proportion reporting high-risk sexual practises continues to rise, especially among HIVpositive MSM, as evident from increasing HIV incidence and STI outbreaks among this core-group.

Conclusions Increases in sexual risk behaviour among MSM in Britain have clearly been observed since 2000, however, definitive conclusions regarding changes in heterosexual behaviour are limited until methodologically-comparable data are available from Natsal-3. These new data will enable us to better examine hypotheses regarding changes in the British population's sexual behaviour across time and across the life-course.

\section{S11.2 SEX IN THE UNITED STATES IN THE NEW MILLENNIUM: TEMPORAL TRENDS AMONG MEN AND WOMEN AGED 15-44 YEARS}

doi:10.1136/sextrans-2013-051184.0055

J S Leichliter, L T Haderxhanaj, H W Chesson, S 0 Aral. Centers for Disease Control and Prevention, Atlanta, GA, United States

Background Examining national trends in sexual behaviour can aid in the understanding of STD trends. We examined trends in sexual behaviour, focusing on sub-populations most impacted by STDs in the US.

Methods We used data from the 2002 and 2006-10 National Survey of Family Growth (NSFG), a multi-stage survey nationally representative of men and women aged $15-44$ years living in the United States (US). The sample sizes and response rates for the surveys were 12, 571 (79\%) in 2002 and 22,682 (77\%) in 2006-10. Sexual behaviours included in this analysis were predominantly from audio computer assisted self-interview and were compared by several demographics, separately by sex. Data were weighted to represent the US population and data analyses accounted for the multi-staged sampling procedures used by NSFG.

Results Sexual behaviours with opposite- and same-sex partners were frequently similar in 2002 and 2006-10. Of women who ever had vaginal sex, there was no change in the average number of partners in the past 12 months (1.21 in 2002, 1.11 in 2006-10); however there was a slight decrease over time for Hispanic and black women and a slight increase among women in the non-Hispanic other category. Findings for men were similar except for a slight increase in partners among white men. Overall, HIV-related risk with oppositesex partners decreased from 2002 to 2006-10. Specifically, exchanging sex for money or drugs significantly decreased among women $(2.0 \%$ to $0.7 \%, p<0.05)$ and men $(2.6 \%$ to $1.3 \%, p<0.05)$. Finally, the average number of male partners decreased among sexually active men who have sex with men (MSM) from 2.9 in 2002 to 2.3 in 2006-10 ( $\mathrm{p}<0.05)$. Specific HIV risk also declined among MSM.
Conclusion Preliminary findings suggest that behaviours have not changed much during this time; however, we did identify shifts in behaviours among sub-populations.

\section{S11.3 SWITZERLAND: NATIONAL TRENDS IN SEXUAL BEHAVIOUR IN THE CONTEXT OF HIV/STI BEHAVIOURAL SURVEILLANCE 1987-2012}

doi:10.1136/sextrans-2013-051184.0056

F Dubois-Arber, A Jeannin, S Lociciro, R Bize, B Spencer, J Gervasoni. Institute of Social and Preventive Medicine (IUMSP), Lausanne University Hospital, Lausanne, Switzerland

Background National trends in sexual behaviour have been assessed mainly in the context of the HIV related behavioural surveillance system set up in Switzerland between 1987 and 1992.

Methods Several populations are included in the system. Repeated surveys have been regularly conducted among the general population and youth, men having sex with other men (MSM), injecting drug users (IDU). Data on sexual behaviour are regularly recorded among people living with HIV/Aids (PLWHA) included in the Swiss HIV Cohort.

Results The main trends observed are :

In young adults (aged 18-20):

- a steady increase in the proportion of sexually active at age 17

- a stable median number of partners with a recent increase in the proportion of multipartners;

- a high and stable level of condom use among multipartners.

Among MSM:

- an increase in the number of partners and a steady increase in unprotected anal intercourse since 1997, after a period of decreasing trends.

Among IDU:

- a low and stable use of condoms with stable partners;

- a high and stable use of condoms with occasional and paying partners (only among women) with a possible recent decreasing trend.

Among PLWHA: a high use of condoms with all types of partners with a recent decrease.

Conclusions The behavioural surveillance system in place allowed to assess various trends in sexual behaviour in several populations such as: long term trends regarding sexual debut, stable trends and recent changes regarding different indicators of sexual activity in the general population, IDU and PLWHA, inversion of trends in sexual activity and condom use among MSM.

\section{S.12 - STD vaccines and correlates of immunity}

\section{S12.1 HUMAN PAPILLOMAVIRUS VACCINES - CORRELATES OF PROTECTION ARE NOT DEFINED}

doi:10.1136/sextrans-2013-051184.0057

\section{A Williamson. University of Cape Town, Cape Town, South Africa}

Specific types of human papillomavirus (HPV) are causally associated with cervical cancer, with at least $99 \%$ of cervical cancers having detectable HPV DNA. Other cancers that also have an association with HPV include anogenital and oropharyngeal malignancies affecting both males and females. Over two thirds of these cancers are associated with HPV types 16 and 18, which are the high-risk types targeted by the HPV vaccines Gardasil and Cervarix. Gardasil also protects from infection with HPV-6 and HPV-11, which cause genital warts and recurrent respiratory papillomatosis. The vaccines are 